\title{
P-branes and the field theory limit
}

\author{
Jan Pieter van der Schaar* \\ Institute for Theoretical Physics, University of Groningen \\ Nyenborgh 4, 9747 AG Groningen, The Netherlands \\ E-mail: 'J.P. van.der.Schaar@phys.rug.nl'
}

Abstract: We investigate Domain-Wall (DW)/Quantum Field Theory (QFT) correspondences in various dimensions. Our general analysis does not only cover the well-studied cases in ten and eleven dimensions but also enables us to discuss DW/QFT correspondences in lower dimensions. New examples include ' $\mathrm{d}$-branes' in six dimensions preserving 8 supersymmetries.

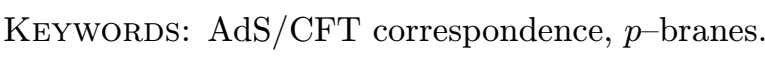

\section{Introduction}

Anti-de Sitter (AdS) supergravity has attracted much attention due to the conjectured correspondence to a conformal field theory (CFT) on the

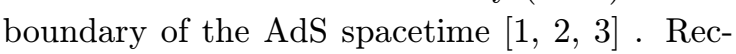
ognizing that this correspondence has its roots in the special properties of D-branes, this correspondence was soon extended to include (most of) the non-conformal ten-dimensional $\mathrm{D} p$-branes [4], general conjecture that there exists a correspondence between Domain-Wall (DW) supergravity and (Yang-Mills) quantum field theory (QFT). This can also be understood by noting that AdS spacetimes are special examples of the more general DW spacetimes [i];]. In this talk we will ex-

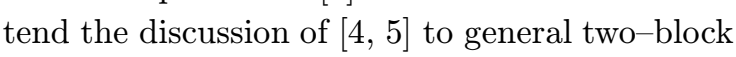
$p$-branes in various dimensions.

In Section 2 we will derive the near-horizon geometry of a generic two-block $p$-brane and in Section 3 we discuss the low energy field theory limit and restrict ourselves to (intersections of) D-branes. We will end that Section by focusing on a six-dimensional example and in Section 4 conclude with a summary and discussion.

* Based on work done in collaboration with Klaus Behrndt, Eric Bergshoeff and Rein Halbersma.

\section{2. $P$-brane near-horizon geometries}

Our starting point is the $D$-dimensional action

$$
\begin{aligned}
S(D, a, p) & =\int \mathrm{d}^{D} x \frac{\sqrt{-g}}{2 \kappa_{D}^{2}}\left[-R-\frac{4}{D-2}(\partial \phi)^{2}\right. \\
& \left.-\frac{g_{s}^{2 k}}{2(d+1) !}\left(\frac{e^{\phi}}{g_{s}}\right)^{a} F_{d+1}^{2}\right], \quad(2.1)
\end{aligned}
$$

which contains three independent parameters: the target spacetime dimension $D$, the dilaton coupling parameter $a$ and a parameter $p$ specifying the rank $D-p-2$ of the field strength $F$. The parameter $k$ is given by

$$
k(D, a, p)=\frac{a}{2}+2 \frac{p+1}{D-2} .
$$

We have furthermore introduced two useful dependent parameters $d$ and $\tilde{d}$ which are defined by

$$
\left\{\begin{array}{l}
d=p+1 \quad \text { wv dimension } \\
\tilde{d}=D-p-3 \quad \text { dual wv dimension }
\end{array}\right.
$$

We next consider the following class of diagonal "two-block" $p$-brane solutions (using the Einstein frame $)^{1}$ :

$$
\begin{aligned}
\mathrm{d} s^{2} & =H^{-\frac{4 \tilde{d}}{(D-2) \Delta}} \mathrm{d} x_{d}^{2}+H^{\frac{4 d}{(D-2) \Delta}} \mathrm{d} x_{\tilde{d}+2}^{2}, \\
e^{\phi} & =g_{s} H^{\frac{(D-2) a}{4 \Delta}},
\end{aligned}
$$

\footnotetext{
${ }^{1}$ For later convenience, we give the solution in terms of the magnetic potential of rank $D-p-3$. The $p$-brane solution is electrically charged with respect to the $p+1-$ form potential.
} 
$g_{s}^{(2-k)} \tilde{F}_{m_{1} \cdots m_{\tilde{d}+1}}= \pm \sqrt{\frac{4}{\Delta}} \epsilon_{m_{1} \cdots m_{\tilde{d}+1} m} \partial_{m} H$

where

$$
g_{s}^{(2-k)} \tilde{F}=\left(\frac{e^{\phi}}{g_{s}}\right)^{a} g_{s}^{k *} F .
$$

We use a constant, i.e. metric independent, LeviCivita tensor. Furthermore, $g_{s}=e^{\phi(H=1)}$ and $\Delta$ is defined by $\left[\begin{array}{c}1 \\ 1\end{array}\right.$

$$
\Delta=\frac{1}{8}(D-2) a^{2}+\frac{2 d \tilde{d}}{D-2},
$$

which is invariant under reductions and oxidations (in the Einstein frame). The function $H$ is harmonic over the $\tilde{d}+2$ transverse coordinates and, assuming that

$$
\tilde{d} \neq-2,0
$$

(i.e. no constant or logarithmic harmonic) this harmonic function is given by

$$
H=1+\left(\frac{r_{0}}{r}\right)^{\tilde{d}} .
$$

Here $r_{0}$ is an integration constant with the dimensions of length. It is related to the mass and charge of the $p$-brane a follows. The mass $\tau_{p}$ per unit $p$-volume is given by the ADM-formula:

$$
\begin{aligned}
\tau_{p} & =\frac{1}{2 \kappa_{D}^{2}} \int_{\partial M^{D-p-1}} d^{D-p-2} \Sigma^{m}\left(\partial^{n} h_{m n}-\partial_{m} h_{b}^{b}\right) \\
& =\frac{2(D-p-3)}{\Delta \kappa_{D}^{2}} r_{0}^{D-p-3} \Omega_{D-p-2} .
\end{aligned}
$$

On the other hand, the charge $\mu_{p}$ per unit $p$ volume is given, in terms of the same integration constant $r_{0}$, by the Gauss-law formula

$$
\begin{aligned}
\mu_{p} & =\frac{1}{2 \kappa_{D}^{2}} \int\left(d^{D-p-2} \Sigma\right)^{m_{1} \cdots m_{\tilde{d}+1}} g_{s}^{(2-k)} \tilde{F}_{m_{1} \cdots m_{D-p-}} \\
& = \pm \sqrt{\frac{\Delta}{4}} \tau_{p}
\end{aligned}
$$

Hence, the $p$-brane solution satisfies the BPS bound

$$
\tau_{p}=\sqrt{\frac{4}{\Delta}}\left|\mu_{p}\right|
$$

To derive an expression for $r_{0}$ in terms of the string parameters $g_{s}$ and $\ell_{s}$, which fixes the scaling of $H$, one must add a source term to the supergravity bulk action. Using the no-force condition $^{2}$ and the fact that in the string frame the

\footnotetext{
${ }^{2}$ Alternatively, one can use a scaling argument, see Appendix B of [10].
}

electric $(p+1)$-form potential $C_{p+1}$ is proportional to $g_{s}^{-k}$, which follows from the action (2.1) , we find that

$$
\tau_{p} \sim \frac{1}{\ell_{s}^{p+1} g_{s}^{k}}
$$

Comparing with (2.9i) and using $\kappa_{D} \sim l_{s}^{D-2} g_{s}^{2}$ we deduce that, for a single brane,

$$
\left(\frac{r_{0}}{\ell_{s}}\right)^{\tilde{d}} \sim g_{s}^{2-k} .
$$

The two-block solutions (2.4) include (supersymmetric) domain-wall spacetimes. They correspond to the case $\tilde{d}=-1, \varepsilon=-1$ and $r_{0}=1 / m$. The solutions also include the known branes in ten and eleven dimensions (M2, M5, D $p, \mathrm{~F} 1$, NS5 etc.) as well as branes in lower dimensions. If the branes under consideration preserve any supersymmetries we can set $[\overline{8} \bar{q}, \overline{9}, \bar{n}$

$$
\Delta=\frac{4}{n}
$$

where generically $32 / 2^{n}$ is the number of unbroken supersymmetries.

We now consider the limit for which the constant part in the harmonic function is negligible. We make a co-ordinate transformation and go to the dual frame

$$
\left(\frac{r_{0}}{r}\right)=e^{-\lambda / r_{0}} \quad g_{*}=e^{\left(\frac{a}{\tilde{d}}\right) \phi} g_{E} .
$$

After these manipulations we can write the nearhorizon metric as

$$
\begin{aligned}
\mathrm{d} s_{*}^{2} & =e^{-2\left(1-\frac{2 \tilde{d}}{\Delta}\right) \lambda / r_{0}} \mathrm{~d} x_{d}^{2}+\mathrm{d} \lambda^{2}+r_{0}^{2} \mathrm{~d} \Omega_{\tilde{d}+1}^{2}, \\
\phi & =-\lambda \frac{(D-2) a \tilde{d}}{4 \Delta r_{0}},
\end{aligned}
$$

which generically (when $1-\frac{2 \tilde{d}}{\Delta} \neq 0$ ) describes an $A d S_{d+1} \otimes S^{\tilde{d}+1}$ geometry and a linear (in $\lambda$ ) dilaton background. Another useful and standard parametrisation, when $1-\frac{2 \tilde{d}}{\Delta} \neq 0$, can be obtained by introducing a radial parameter $u$ with dimensions of mass

$$
u=\frac{r^{\beta}}{r_{0}^{\beta+1}}, \quad \beta=\frac{2 \tilde{d}}{\Delta}-1
$$

and the metric after a rescaling of $x_{d} \rightarrow \beta^{-2} x_{d}$, can be written as

$$
\mathrm{d} s_{*}^{2}=r_{0}^{2}\left[\beta^{-2}\left\{u^{2} \mathrm{~d} x_{d}^{2}+\left(\frac{\mathrm{d} u}{u}\right)^{2}\right\}+\mathrm{d} \Omega_{\tilde{d}+1}^{2}\right] .
$$


Reducing over the $\tilde{d}+1$ angular variables of the sphere we end up with a gauged supergravity in $d+1$ dimensions supporting a domain-wall solution. The precise relation between the parameters of the domain-wall supergravity action and its solution in terms of those of the $p$-brane

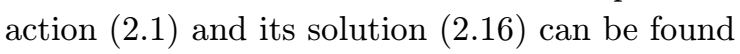
in [1]

Summarizing, in this Section we showed that in the dual frame, defined by $(\overline{2} . \overline{1})$, all $p$-branes solutions (2.4) have a near-horizon background $\mathrm{DW}_{d+1} \otimes S^{\bar{d}+1}$. The domain-wall metric has all the isometries of an AdS space. These isometries are broken in the full background because of the presence of a non-trivial dilaton.

\section{The field theory limit}

In this section we will set up the framework for the DW/QFT duality similar to the analysis of [Fin but for arbitrary dimensions. As we showed in the previous section, the near-horizon geometry of a general $p$-brane in the dual frame is equivalent to that of a non-dilatonic $p$-brane. It is therefore natural to assume that the duality might be extended. The presence of the dilaton turns the AdS background into a DW background and the conformal field theory into a nonconformal QFT, hence the name DW/QFT duality. In the following we will restrict ourselves to (intersecting) D-branes reduced over all relative transverse directions ${ }^{3}$, giving a particular dilatonic $p$-brane solution in lower dimensions. This restriction to $\mathrm{D}$-branes (or equivalently $k=1$ ), as one might expect, is necessary to obtain nonsingular dilaton expressions after taking the limit [1010.].

For the field theory to be non-trivial we need that at least one coupling constant is fixed in the low energy limit $l_{s} \rightarrow 0$. Based on dimensional analysis and the scaling of the effective tension with $g_{s}(2.12)$ we can deduce that the $p$-brane worldvolume field theory has a ('t Hooft) coupling constant $g_{f}^{2}$ which can be written as follows:

$$
g_{f}^{2}=c_{p} N g_{s}^{k} l_{s}^{x}
$$

\footnotetext{
${ }^{3}$ This also means that these intersecting D-brane configurations are delocalized.
}

Here $N$ denotes the number of stacked branes and the scaling of the coupling constant with $g_{s}$ is as the inverse tension $\tau_{p}{ }^{-1}$. The undetermined numerical factor $c_{p}$ and the parameter $x$ depend on the specific field theory under consideration. For a rank $q$ tensor field theory in $p+1$ dimensions, $x$ is in fact equal to $p-2 q-1$. Depending on whether $x$ is positive or negative, the factor

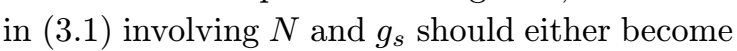
large or small in the low energy limit in order to keep $g_{f}^{2}$ fixed. In order for the theory to decouple from the bulk supergravity we will assume that $g_{s} \ll 1$ (so $\kappa_{D} \rightarrow 0$ in the low energy limit) and therefore we take $N \rightarrow \infty$ when $g_{s} N$ has to become large to fix $g_{f}$.

As explained in [1 1, there are two natural energy scales we can keep fixed in the low energy limit, depending on how we probe the collection of D-branes. They concluded that probing a collection of D-branes with supergravity fields is the natural thing to do in the context of holography, instead of probing the system with another Dbrane. In [5] it was observed that in the dual frame, where the metric describes an AdS spacetime, the radial parameter $u\left(\mathbf{1}^{2} . \overline{1} \overline{7}\right)$ in the metric (i2.18) naturally corresponds to the energy scale of a supergravity field probe. Clearly this observation can now be extended to all two-block $p$-branes and we will use the energy scale $u$ as the fixed holographic energy scale.

We demand the two different energy scales to be related by some fixed quantity (in the low energy field theory limit). After all, using one or the other probe should not make a (singular) difference. As is explained in [10 10 this gives the following constraint on $x$

$$
x=\Delta-\tilde{d} .
$$

This tells us which coupling constant in the field theory we should keep fixed. When $\Delta=4$ and $\tilde{d}=7-p$ we find that $x=3-p$, which is the scaling of a Yang-Mills coupling constant appropriate for D-branes in $D=10\left[\begin{array}{ll}1 \\ 1\end{array}\right]$ and $D=6$ we find $x=1-p$, which is the appropriate scaling of a scalar field coupling constant (and the conformal $p=1$ case related to the D1D5 intersection was already discussed in [i1]

Rewriting the supergravity background solution in terms of the fixed field theory quanti- 
ties and taking the low energy limit gives us the near-horizon metric ( $\left(\overline{2} . \overline{1} \bar{q}_{1}^{\prime}\right)$ (all $l_{s}$ dependence disappears) and the non-trivial dilaton becomes

$$
e^{\phi}=\frac{1}{N}\left[\left(g_{f}^{2}\right)^{1 / x} u \beta\left(\frac{d_{p}^{1 / \tilde{d}}}{c_{p}^{1 / x}}\right)\right]^{\frac{-(D-2) a}{8}\left(\frac{\beta+1}{\beta}\right)} .
$$

So the complete background solution is well behaved everywhere except for the special points $u=0, \infty$ where the dilaton either vanishes or blows up. The conjecture is that there is a correspondence between string theory on this DW background and the $p$-brane theory in the low energy field theory limit. We note that (as in $D=10)$ string quantum effects are $1 / N$ effects and a supergravity approximation can be used when the string coupling and the (string) spacetime curvature are both small. However, the conformal invariance which in the AdS/CFT duality facilitates computations in the strongly coupled field theory is now broken so that any direct check of a DW/QFT duality is ruled out. Let us end this section by discussing the sixdimensional d0-brane example. For details about other new examples (like the D8-brane in $D=10$ and the d4-brane in $D=6^{4}$ ) we refer to [1 101$]$.

In $D=6$ we keep the scalar coupling constant fixed. This also means that the Yang-Mills coupling constant diverges. Assuming we are on the Higgs branch, giving the gauge fields masses proportional to the diverging Yang-Mills coupling constant, the massive gauge fields will decouple and we are left with Higgs branch physics only (also assuming that the Higgs branch decouples from the Coulomb branch as claimed in

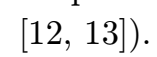

Let us investigate where we can trust the perturbative field theory and the supergravity approximation. Clearly the perturbative field theory has become a quantum mechanics model and the dimensionless effective coupling constant governing the perturbative expansion in this case is defined as

$$
g_{\mathrm{eff}}^{2}=g_{f}^{2} u \text {. }
$$

\footnotetext{
${ }^{4}$ These cases are special because they require $N \rightarrow$ $\infty$ to decouple gravity and the correspondence seems to indicate that perturbative QFT and DW supergravity are valid in the same energy regime, which at first sight seems contradictory.
}

Small curvature can be translated into the following constraint

$$
\tau_{D}=\left(d_{p} N e^{\phi}\right)^{2 / 3} \gg 1
$$

This is the effective tension of closed strings in the dual frame and a particle (supergravity) approximation is valid when this tension is large. We denote the crossover from a supergravity approximation to a string theory by the point $u_{N}$ and the crossover from a perturbative quantum mechanics model to a strongly coupled quantum mechanics model by $u_{g}$.

In the case of the d0-brane the supergravity approximation can be used in the following IR energy regime

$$
u_{N}=g_{f}^{2} N^{\frac{-4}{3}} \ll u \ll u_{g}=g_{f}^{2},
$$

which can only be satisfied for large $N$. In the UV we can use perturbative field theory

$$
u \gg u_{g}
$$

which in this case reduces to a quantum mechanics model. We therefore find the typical $D W / Q F T$ behavior that the supergravity regime and the perturbative field theory regime do not overlap, avoiding inconsistencies. In the far IR, when

$$
u \ll u_{N},
$$

the string coupling becomes large and we could try to use an S-dual description. The different regimes are plotted in Figure 1.

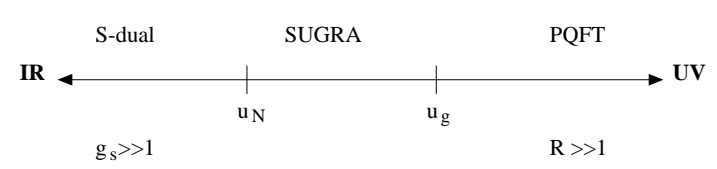

Figure 1: Different regimes in the energy plot for d0-branes with $N \gg 1$.

The d0-brane is related to the D4-brane by considering a IIA compactification on a $K 3$ manifold. It is conjectured (and by now well established) that Type IIA superstring theory on a K3 manifold is $\mathrm{S}-$ dual to Heterotic superstring theory on a $T^{4}[1-14]$. On the Heterotic side the S-dual soliton solution would be a fundamental state $(k=0)$ and has a curvature singularity at $u=0$, so a supergravity approximation will 
not make sense. The situation resembles the D1brane case in $D=10$ Type IIB theory. There the curvature singularity in the S-dual F1-brane solution was resolved by the strong coupling conformal fixed point of the $(1+1)$-dimensional gauge theory. It is suggestive to propose the occurrence of a similar phenomenon in this case. It would therefore be interesting to determine the strongly coupled IR limit of the corresponding quantum mechanics model.

Let us remark however that in the context of the DW/QFT correspondence the quantum me-

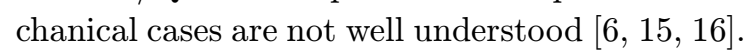
This has to do with the fact that quantum mechanics does not have "internal" worldline dynamics and only when we take the 0-branes apart do we expect to obtain a dynamical model. However, when we take the 0 -branes apart it is not clear what the corresponding dual supergravity background should be.

\section{Conclusions and discussion}

At the end of this talk let us make the following remarks. We did not present a detailed investigation of the $p$-brane worldvolume field theories. Our discussion was focussed on the $p$-brane geometries in the field theory limit and the search for well-defined supergravity regions. We showed that the near-horizon geometry of the $D=6$ d0-brane indeed has regions where a supergravity approximation seems valid and the analysis is strikingly similar to that of the $D=10 \mathrm{D} p$ branes with $p<3$.

We did make some general remarks on the nature of the field theory, which is governed by scalar dynamics, presumably in the Higgs branch of the $p+1$-dimensional gauge theory, consisting of supersymmetry vector multiplets and hypermultiplets. This suggests a possible relation with Matrix theory descriptions of the M5-brane [1 13 n] where a similar limit is described. It would be interesting to pursue this connection further.

We want to point out that other work was done on localized $\mathrm{D} p$-brane intersections and the field theory limit $\left[\overline{1} \overline{7}_{1}^{\prime}, \overline{1} \overline{8}_{1}^{\prime}, \overline{1}_{2}^{\prime \prime}\right]$. In these investigations a limit is considered taking one into the near-horizon geometry of the lower-dimensional D-brane in the intersection and the dual field theory should then also be the one living on the lower-dimensional D-brane. Although the field theory limit in that case fixes the Yang-Mills coupling constant, there could be a connection with the results presented here in the sense that both investigations start off with the same intersecting D-brane system.

\section{Acknowledgements}

The work reported here is based on hep-th/9907006. I thank my collaborators Klaus Behrnd, Eric Bergshoeff and Rein Halbersma for many discussions. This work is part of the research program of the "Stichting voor Fundamenteel Onderzoek der Materie" (FOM) and also supported by the European Commission TMR programme ERBFMRX-CT96-0045, in which J.P. v.d. S. is associated to the University of Utrecht.

\section{References}

[1] J. Maldacena, The large $n$ limit of superconformal field theories and supergravity, Adv. Theor. Math. Phys. 2 (1998) 231, hep-th/9711200i].

[2] E. Witten, Anti-de sitter space and holography, Adv. Theor. Math. Phys. 2 (1998) 253-291, hep-th/9802150i].

[3] S. S. Gubser, I. R. Klebanov, and A. M. Polyakov, Gauge theory correlators from non-critical string theory, Phys. Lett. B428 (1998) 105, [hep-th/9802109']

[4] N. Itzhaki, J. M. Maldacena, J. Sonnenschein, and S. Yankielowicz, Supergravity and the large $n$ limit of theories with sixteen supercharges, Phys. Rev. D58_(1998) 046004, hep-th/9802042".

[5] H. J. Boonstra, K. Skenderis, and P. K. Townsend, The domain wall/qft correspondence, JHEP 01 (1999) 003, hep-th/98071377.

[6] O. Aharony, S. S. Gubser, J. Maldacena, H. Ooguri, and Y. Oz, Large n field theories, string theory and gravity, hep-th/9905111.'.

[7] H. Lu, C. N. Pope, and P. K. Townsend, Domain walls from anti-de sitter spacetime, Phys. Lett. B391 (1997) 39-46, hep-th/9607164i . 
[8] H. Lu, C. N. Pope, E. Sezgin, and K. S. Stelle, Dilatonic p-brane solitons, Phys. Lett. B371 (1996) 46-50, [hep-th/9511203'.

[9] H. Lu, C. N. Pope, E. Sezgin, and K. S. Stelle, Stainless super_p-branes, Nucl. Phys. B456 (1995) 669, ihep-th/9508042"

[10] K. Behrndt, E. Bergshoeff, R. Halbersma, and J. P. van der Schaar, On domain wall / qft dualities in various dimensions, hep-th/9907006.

[11] A. W. Peet and J. Polchinski, Uv/ir relations in ads dynamics, Phys. Rev. D59 (1999) 065011, hep-th/9809022].

[12] E. Witten, On the conformal field theory of the higgs branch, JHEP 07 (1997) 003, hep-th/9707093.

[13] O. Aharony, M. Berkooz, S. Kachru, N. Seiberg, and E. Silverstein, Matrix description of interacting theories in six dimensions, Adv. Theor. Math. Phys. 1 (1998) 148-157, hep-th/9707079].

[14] E. Witten, String theory dynamics in various dimensions, Nucl. Phys. B443 (1995) 85-126, hep-th/9503124].

[15] J. Maldacena, J. Michelson, and A. Strominger, Anti-de sitter fragmentation, JHEP 02 (1999) 011, hep-th/9812073".

[16] R. Britto-Pacumio, J. Michelson, A. Strominger, and A. Volovich, Lectures on superconformal quantum mechanics and multiblack hole moduli spaces, hep-th/9911066.

[17] S. Ferrara, A. Kehagias, H. Partouche, and A. Zaffaroni, $A d s(6)$ interpretation of $5 d$ superconformal field theories, Phys. Lett. B431 (1998) 57-62, hep-th/9804006.].

[18] A. Brandhuber and Y. Oz, The d4-d8 brane system and five dimensional fixed points, Phys. Lett. B460 (1999) 307, hep-th/9905148in.

[19] O. Pelc and R. Siebelink, The d2-d6 system and a fibered ads geometry, hep-th/9902045. 